\title{
Incidence of Inadvertent Intravascular Injection during CT Fluoroscopy-Guided Epidural Steroid Injections
}

(DP.G. Kranz, T.J. Amrhein, and L. Gray

\begin{abstract}
BACKGROUND AND PURPOSE: Inadvertent intravascular injection during epidural steroid injection can result in complications and has been investigated previously with conventional fluoroscopy, but not CT fluoroscopy. The purpose of this study was to determine the incidence of intravascular injections recognized during CT fluoroscopy-guided epidural steroid injection.
\end{abstract}

MATERIALS AND METHODS: We retrospectively reviewed 575 consecutive CT fluoroscopy-guided epidural steroid injections. Procedures were assessed to determine the incidence of intravascular injection. Cases positive for intravascular injection were classified on the basis of anatomic location, distance from the needle tip, washout pattern, and presence of combined epidural and vascular injection. Cases were also graded as either venous or arterial by using a 5-point scale.

RESULTS: Intravascular injection was observed in $26 \%$ of cervical transforaminal epidural steroid injections $(7 / 27), 9 \%$ of cervical interlaminar epidural steroid injections (4/47), 8\% of lumbar transforaminal epidural steroid injections (22/275), and $2 \%$ of lumbar interlaminar epidural steroid injections (4/222). Vessels were most commonly identified close to the needle, but in $30 \%$ of cases, they were visualized in the anterior paraspinal soft tissues remote from the needle. Washout was most commonly delayed (86\%), though rapid washout occurred in $14 \%$ of cases. Simultaneous epidural and vascular injections occurred in $32 \%$ of cases. Most visualized vessels were venous, but 2 cases were classified as probably arterial.

CONCLUSIONS: Intravascular injections can be detected with CT fluoroscopy. The incidence in our study was similar to that in previous reports using conventional fluoroscopy. Technical factors such as the "double-tap" on CT fluoroscopy following contrast injection, assessment for discordance between injected and visualized contrast volume, and maintenance of an appropriate FOV facilitate the detection of such events.

ABBREVIATIONS: CTF = CT fluoroscopy; ESI = epidural steroid injection; ILESI = interlaminar epidural steroid injection; TFESI = transforaminal epidural steroid injection

E pidural steroid injections (ESIs) are a commonly performed intervention for patients with degenerative conditions of the spine. ${ }^{1}$ Recent attention to rare but potentially catastrophic side effects associated with ESIs has resulted in an increased focus on maximizing the safety of these injections. ${ }^{2,3}$ It is thought that most severe adverse events are vascular, the result of either embolic phenomena during injection of particulate steroid into arteries that supply the spinal cord or brain, or direct vascular injury during needle placement. ${ }^{4}$

The incidence of unintended intravascular needle placement

Received September 5, 2014; accepted after revision October 21

From the Department of Radiology, Duke University Medical Center, Durham, North Carolina.

Please address correspondence to Peter G. Kranz, MD, Department of Radiology, Box 3808, Duke University Medical Center, Durham, NC 27710; e-mail: peter.kranz@duke.edu

http://dx.doi.org/10.3174/ajnr.A4219 during procedures performed with conventional fluoroscopy has been previously reported in multiple investigations. ${ }^{5-9}$ Several authors have advocated that real-time fluoroscopy or digital subtraction angiography be used when epidural injections are performed, to maximize the detection of intravascular injections. ${ }^{10-12}$ However, CT fluoroscopy (CTF) guidance, which does not use real-time fluoroscopy or DSA but does offer superior contrast resolution compared with fluoroscopy, has been increasingly used by some practitioners when performing epidural injections because it provides the benefits of cross-sectional needle localization and improved visualization of soft-tissue structures. ${ }^{13,14}$ The ability to detect intravascular injections by using CT guidance has not been previously reported, to our knowledge. Nevertheless, it has been assumed by some authors to be inferior to conventional fluoroscopy in this respect. ${ }^{15-17}$

The purpose of this investigation was to report the incidence of intravascular injections detected during CTF-guided epidural 
steroid injections. Additionally, we sought to characterize the appearance of intravascular injections to guide proceduralists unaccustomed to observing these injections with CT.

\section{MATERIALS AND METHODS}

All consecutive CTF-guided ESIs performed by a single proceduralist at 1 outpatient facility during an 18-month period (January 2013 to June 2014) were retrospectively reviewed. The proceduralist was a board-certified radiologist who holds a Certificate of Added Qualification in neuroradiology and has 7 years' experience performing injections under CTF guidance. The investigation was approved by our local institutional review board and is compliant with Health Insurance Portability and Accountability Act regulations.

Cases were identified by review of departmental procedure schedules and were considered on a per-injection basis (ie, if patients underwent $>1$ injection in a single visit, each individual injection was considered a separate case for data analysis).

\section{Injection Technique}

Injections were performed by using intermittent CTF on a single CT scanner (LightSpeed 16; GE Healthcare, Milwaukee, Wiscon$\sin$ ), equipped with a foot pedal used to trigger acquisition of a set of 3 consecutive fluoroscopic images, using a peak voltage of 120 $\mathrm{kV}$ (peak) and a section thickness of $2.5 \mathrm{~mm}$. The tube current and display FOV were selected by the radiologist on a case-by-case basis, depending on the age and body habitus of the patient and the complexity of the local anatomy. In general, the tube current was typically set at 20-60 mA for lumbar injections and approximately 50\% higher for cervical injections. The display FOV was
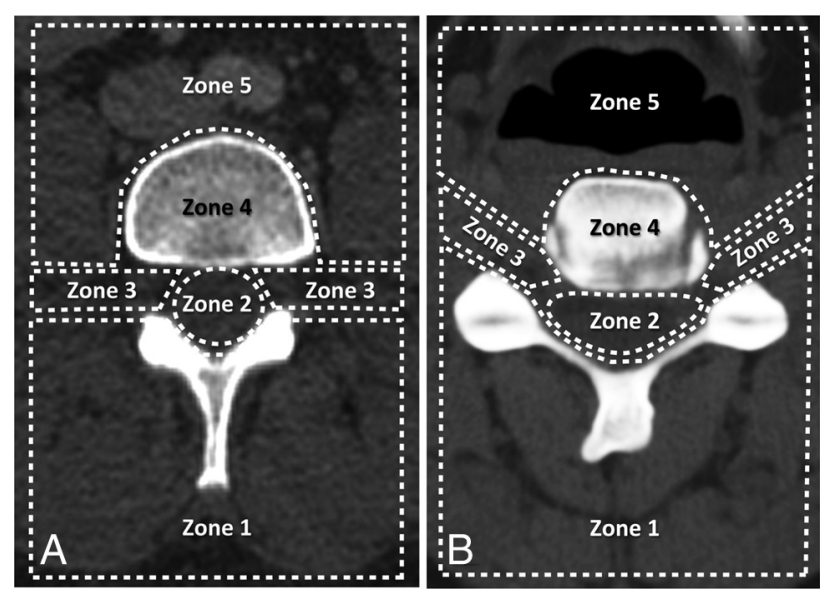

FIG 1. Classification scheme for the location of visualized vessels seen during inadvertent intravascular injection in the lumbar $(A)$ and cervical (B) spine. Regions include the following: posterior paraspinal soft tissues (zone 1), spinal canal (zone 2), foraminal region (zone 3), vertebral body (zone 4), and anterior paraspinal soft tissues (zone 5). selected to encompass, at minimum, the skin surface of the back to the anterior margin of the vertebral body.

Contrast was used for all injections before injection of steroid. For lumbar injections, undiluted iopamidol containing 200$\mathrm{mg} / \mathrm{mL}$ iodine was used (Isovue-M 200; Bracco, Princeton, New Jersey). For cervical injections, a 50\% dilution of iopamidol with preservative-free sterile saline was used to achieve a final iodine concentration of $100-\mathrm{mg} / \mathrm{mL}$ iodine. ${ }^{18}$ The injected contrast volume was approximately $0.2-0.4 \mathrm{~mL}$ for the first injection. Repeat injections using larger volumes of contrast $(0.5-1.0 \mathrm{~mL})$ were used if the first injection was not sufficient due to either poor visualization or suspected vascular washout. After contrast injection, 2 sets of fluoroscopic images were obtained by using a "double-tap" approach, whereby the foot pedal was pressed immediately following the injection to determine the initial contrast pattern and again 2-3 seconds later to obtain a delayed image to evaluate contrast washout. A 22-ga Quincke Point Spinal Needle (BD Medical, Franklin Lakes, New Jersey) was used for all injections. If blood flash was seen in the needle hub, the needle was repositioned before contrast injection. For transforaminal epidural steroid injections (TFESIs), care was taken to position the needle as far posterior in the neural foramen as possible. An anesthetic test dose was performed during cervical TFESI. ${ }^{19}$ Procedures were otherwise performed as previously described. ${ }^{13,20,21}$

\section{Image Analysis}

All consecutive cases of ESIs performed during the study time range were reviewed on a PACS by the radiologist who performed the procedures to identify cases of unintended intravascular injection. Images obtained after contrast injection were compared with preinjection images at the same level to ensure that attenuation related to osseous structures was not misinterpreted as vascular contrast. Findings of cases were considered positive if there was either direct visualization of vascular structures or if there was little-to-no contrast visualized after contrast injection, after checking to ensure the absence of a leak from the connector tubing. Cases that demonstrated intravascular injection were then independently reviewed and confirmed by a second board-certified radiologist who holds a Certificate of Added Qualification in neuroradiology and who has 4 years' experience performing injections under CT fluoroscopy.

Both readers then classified the intravascular contrast in cases positive for it according to its location relative to the spinal canal, distance from the needle tip, and washout pattern. The location of the contrast was categorized into 5 predetermined anatomic regions, as depicted in Fig 1. Multiple locations could be assigned per injection if intravascular contrast was identified in $>1$ zone simultaneously. The rate of contrast washout was assessed by comparing the immediate CTF image with the delayed image and was classified as one of the following: no washout, rapid washout, or delayed washout. "Rapid washout" was defined as a case in which there was complete

Table 1: Assessment criteria for vessel type seen during intravascular injection

\begin{tabular}{cll}
\hline Score & \multicolumn{1}{c}{ Vessel } & \multicolumn{1}{c}{ Suggestive Features } \\
\hline 5 & Definite vein & Flow into a specific, anatomically identifiable venous structure \\
4 & Probable vein & Flow into region of known venous structure, flow predominantly away from spinal canal, delayed washout \\
3 & Indeterminate & Not meeting criteria for other categories \\
2 & Probable artery & Flow into region of known arterial structure, flow predominantly toward the spinal canal, rapid washout \\
1 & Definite artery & Flow into a specific, anatomically identifiable arterial structure \\
\hline
\end{tabular}


Table 2: Frequency of intravascular injection by anatomic zone ${ }^{a}$

\begin{tabular}{|c|c|c|c|c|c|c|c|}
\hline \multirow[b]{2}{*}{ Injection Type } & \multicolumn{6}{|c|}{ Anatomic Zone of Identified Vessel } & \multirow[b]{2}{*}{ No. of Injections } \\
\hline & 1 & 2 & 3 & 4 & 5 & 0 & \\
\hline Lumbar ILESI & $25 \%$ & $100 \%$ & $25 \%$ & $0 \%$ & $0 \%$ & $0 \%$ & 4 \\
\hline Lumbar TFESI & $14 \%$ & $18 \%$ & $86 \%$ & $5 \%$ & $36 \%$ & $0 \%$ & 22 \\
\hline Cervical ILESI & $0 \%$ & $100 \%$ & $50 \%$ & $0 \%$ & $50 \%$ & $0 \%$ & 4 \\
\hline Cervical TFESI & $29 \%$ & $0 \%$ & $71 \%$ & $0 \%$ & $14 \%$ & $14 \%$ & 7 \\
\hline
\end{tabular}

${ }^{a}$ Percentages across each injection type may sum to $>100 \%$ because vessels may be visualized in $>1$ location simultaneously.
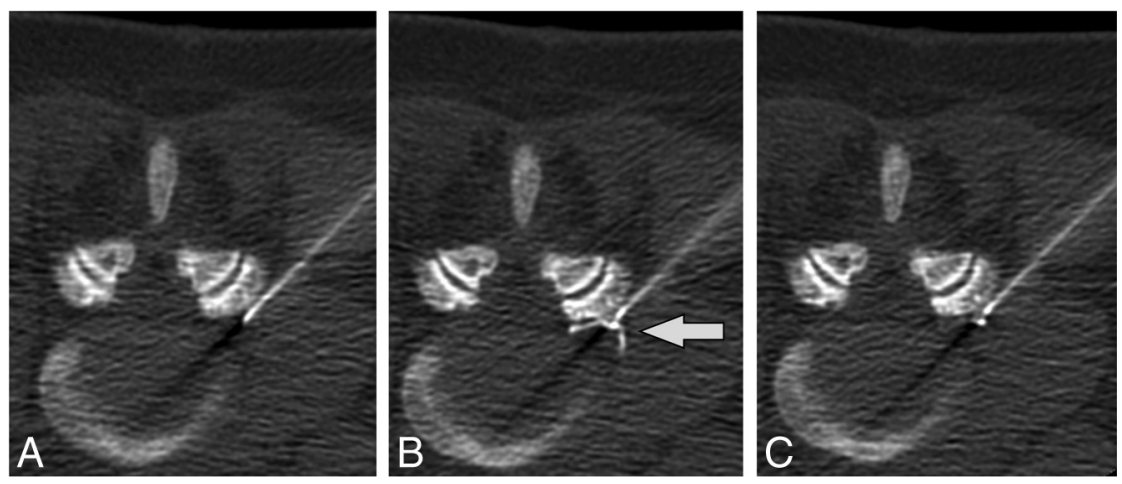

FIG 2. Intravascular injection during lumbar TFESI. Preinjection (A), immediate postinjection (B), and delayed postinjection $(C)$ images demonstrate a vessel in the left foraminal zone (arrow) that washes out on the delayed image.
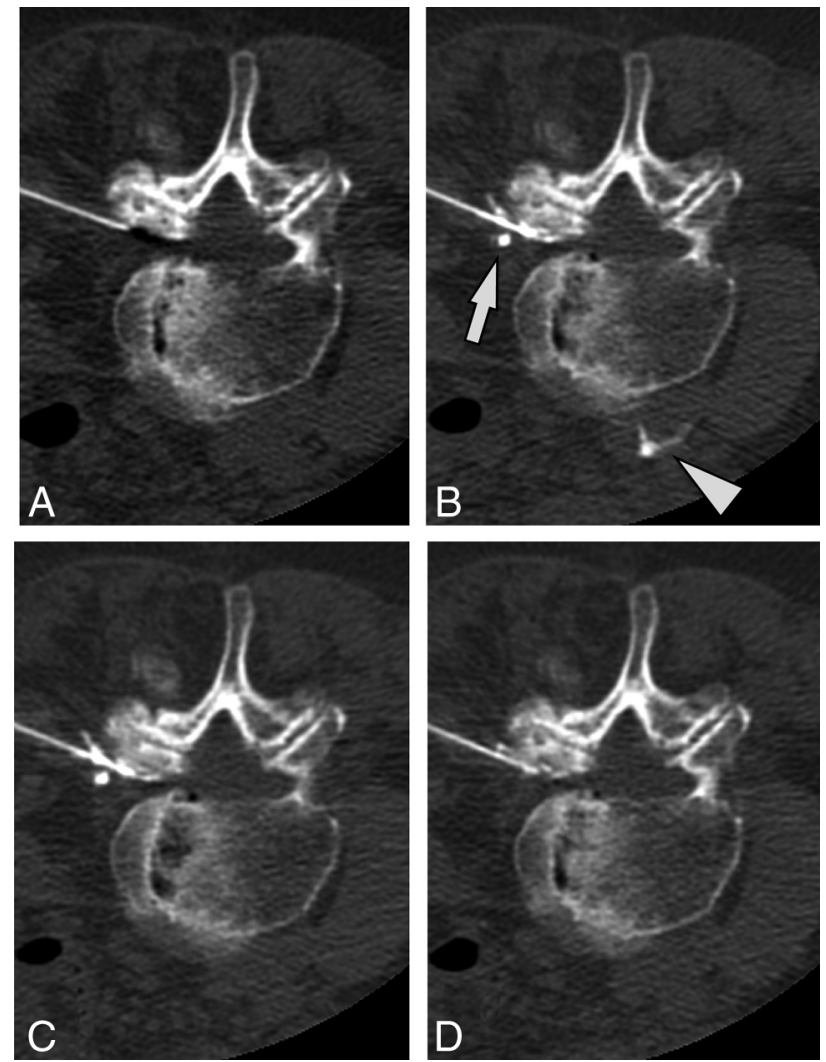

FIG 3. A case of intravascular injection is demonstrated on preinjection $(A)$, immediate postinjection (B), first delayed $(C)$ images, and an additional delayed $(D)$ image. On the immediate postinjection image, contrast is seen in the ascending lumbar vein (arrow) and the inferior vena cava (arrowhead). There is washout of contrast from the inferior vena cava on the first delayed image and from the ascending lumbar vein on the second delayed image. absence of contrast on the immediate image or there was markedly less contrast than expected given the volume injected. "Delayed washout" was defined as a case of vascular opacification in which dense intravascular contrast was seen on the immediate image but it decreased or disappeared on the delayed postinjection images. Distance from the needle tip to the opacified vessel was assigned to 1 of 4 categories: $<1 \mathrm{~cm}$ from needle tip to the vessel, $>1 \mathrm{~cm}$, both $>1 \mathrm{~cm}$ and $<1 \mathrm{~cm}$, or vessel not seen due to immediate washout. Cases positive for intravascular injection were also classified as showing either vascular opacification alone or simultaneous vascular and epidural contrast.

The readers also attempted to classify the opacified vessel as either venous or arterial and provided a confidence level for this assessment based on criteria set forth in the 5-point scale shown in Table 1. Cases in which the readers disagreed were rereviewed by both readers, and a consensus score was determined. Other patient characteristics, including age and sex, were also recorded. For data analysis, sacral TFESIs were included with lumbar injections.

\section{Statistical Analysis}

Statistical analyses were performed by using commercially available software (GraphPad Prism 6 software, Version 6.0b; GraphPad Software, San Diego, California). The Mann-Whitney $U$ test was used to compare differences in age among groups. The Fisher exact test was used to compare differences in sex. $P$ values $<.05$ were considered statistically significant.

\section{RESULTS}

\section{Subject Characteristics}

A total of 575 consecutive epidural injections were identified during the study period. These included 275 lumbar transforaminal injections, 222 lumbar interlaminar injections, 47 cervical interlaminar injections, 27 cervical transforaminal injections, 3 thoracic transforaminal injections, and 1 thoracic interlaminar injection performed during 390 individual patient encounters. The mean patient age for all injections was 63.5 years (range, 22-92 years). Forty-three percent of injections were performed on male patients, and 57\%, on female patients. All injections were technically successful. In all cases in which intravascular injection was identified during the procedure, the needle could be repositioned and the subsequent steroid injection was performed successfully. There were no major complications in any subject.

\section{Incidence of Intravascular Injections}

Intravascular injection was observed in $26 \%$ of cervical TFESIs (7/27), 9\% of cervical interlaminar epidural steroid injections (ILESIs) (4/47), 8\% of lumbar TFESIs (22/275), and $2 \%$ of lumbar 
ILESIs (4/222). No cases of intravascular injection were identified during thoracic epidural injections; however, the study population included only 3 thoracic TFESIs and 1 thoracic ILESI, limiting conclusions regarding incidence, given the small number of cases. The overall rate of intravascular injection for all cases was $6 \%(37 / 575)$. No difference in age $(P=.83)$ or sex $(P=.17)$ was observed among patients with intravascular injection detected compared with those without.

\section{Characteristics of Observed Intravascular Injections}

The locations of vessels identified during intravascular injections are reported in Table 2. The most common location of vessels observed during TFESI was in the foraminal region (zone 3), with $86 \%$ and $71 \%$ of TFESIs positive for intravascular injections in the lumbar and cervical regions, respectively, showing vascular opacification in this region (Fig 2). For cases positive for intravascular injection during ILESI, the spinal canal (region 2) was the most commonly observed location of vascular opacification, seen in $100 \%$ of both cervical and lumbar injections. In $46 \%$ of cases (17/37), vessels were seen in $>1$ region simultaneously. Of note, $30 \%$ of cases showed vascular opacification in the anterior paraspinal soft tissues (zone 5) (Fig 3), including 1 case of a lumbar transforaminal injection in which this was the only location where contrast was seen.

\section{Table 3: Characteristics of identified vascular injections}

\begin{tabular}{lrr}
\hline & $\boldsymbol{n}$ & $\%$ \\
\hline Distance from needle tip to vessel & & \\
$<1 \mathrm{~cm}$ only & 11 & $30 \%$ \\
$>1 \mathrm{~cm}$ only & 2 & $5 \%$ \\
Both $<1 \mathrm{~cm}$ and $>1 \mathrm{~cm}$ & 23 & $62 \%$ \\
$\quad$ Immediate washout (vessel not seen) & 1 & $3 \%$ \\
Combined epidural injection & & \\
$\quad$ Vascular only & 25 & $68 \%$ \\
$\quad$ Epidural + vascular & 12 & $32 \%$ \\
Washout pattern & & \\
$\quad$ None & 0 & $0 \%$ \\
$\quad$ Delayed & 32 & $86 \%$ \\
$\quad$ Immediate & 5 & $14 \%$ \\
Vessel type & & \\
$\quad$ Definite venous $(n=5)$ & 4 & $11 \%$ \\
$\quad$ Probable venous $(n=4)$ & 27 & $73 \%$ \\
$\quad$ Indeterminate $(n=3)$ & 4 & $11 \%$ \\
$\quad$ Probable arterial $(n=2)$ & 2 & $5 \%$ \\
$\quad$ Definite arterial $(n=1)$ & 0 & $0 \%$ \\
\hline
\end{tabular}

Data related to the distance from the needle tip, combined epidural and vascular injection, washout pattern, and suspected vessel type are reported in Table 3. Most commonly, vessels were seen simultaneously at both $>1 \mathrm{~cm}$ and $<1 \mathrm{~cm}$ from the needle tip (62\% of cases) (Fig 4). However, in 2 cases (5\%), vessels were only seen $>1 \mathrm{~cm}$ from the needle tip. In 1 case, immediate washout of contrast resulted in no vessel or epidural contrast being seen despite contrast injection (Fig 5).

Simultaneous epidural and vascular injections were observed in $32 \%(12 / 37)$ with positive results.

Washout of contrast between the initial image and the delayed image was seen in all positive cases. Most commonly, the washout pattern was delayed (86\%, 32/37 cases) (Fig 6). However, rapid washout was observed in $14 \%(5 / 37)$ of cases, indicating rapid flow within the vessel.

Most cases were classified as "definitely venous" or "probably venous," with these 2 categories accounting for $84 \%$ of all recognized vascular injections. Four cases $(11 \%)$ were classified as indeterminate. Two cases $(5 \%)$ of probable arterial injection were identified, both of which occurred during cervical transforaminal injections.

\section{DISCUSSION}

Our investigation demonstrates that intravascular injections can be recognized during CTF-guided epidural injections. We found that intravascular injections were identified most commonly during cervical transforaminal injections, in which they were observed in more than one-quarter of cases. Overall the detection rates for intravascular injections in our study are comparable with or, in some cases, slightly greater than rates previously reported by investigators using conventional fluoroscopy. These findings are important in that they directly contradict claims that the crosssectional nature of CT imaging precludes detection of intravascular injections. ${ }^{15-17}$

Although the incidence of unintended intravascular injections during CTF-guided epidural injections has not been previously reported, to our knowledge, recognition of intravascular injection during fluoroscopically guided procedures by using live fluoroscopy or DSA has received considerable attention in the literature. In a prospective study of 2145 transforaminal injections performed by using live fluoroscopy, $\mathrm{Nahm}$ et $\mathrm{al}^{5}$ found an incidence of intravascular injection of $20.6 \%$ in the cervical region and $6.1 \%$
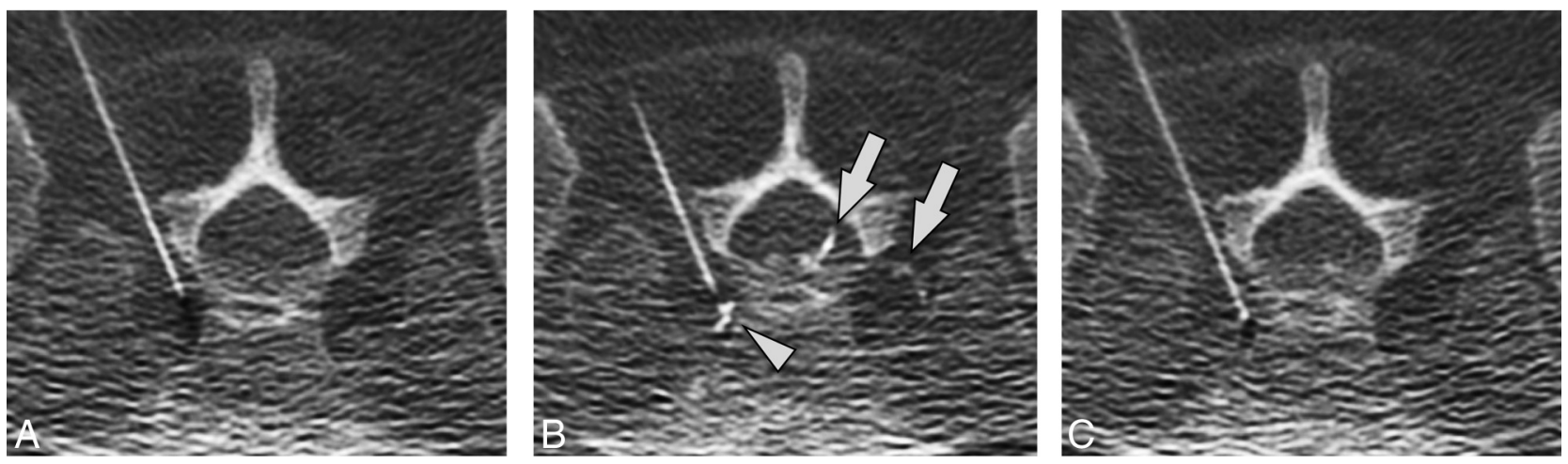

FIG 4. Intravascular contrast seen both close to and more remote from the needle tip. Preinjection ( $A$ ), immediate postinjection ( $B$ ), and delayed postinjection $(C)$ images show intravascular contrast at the needle tip (arrowhead) and further away (arrows) in the sacral epidural venous plexus. Note that these vessels are not seen on the delayed washout image. No epidural contrast is seen. 

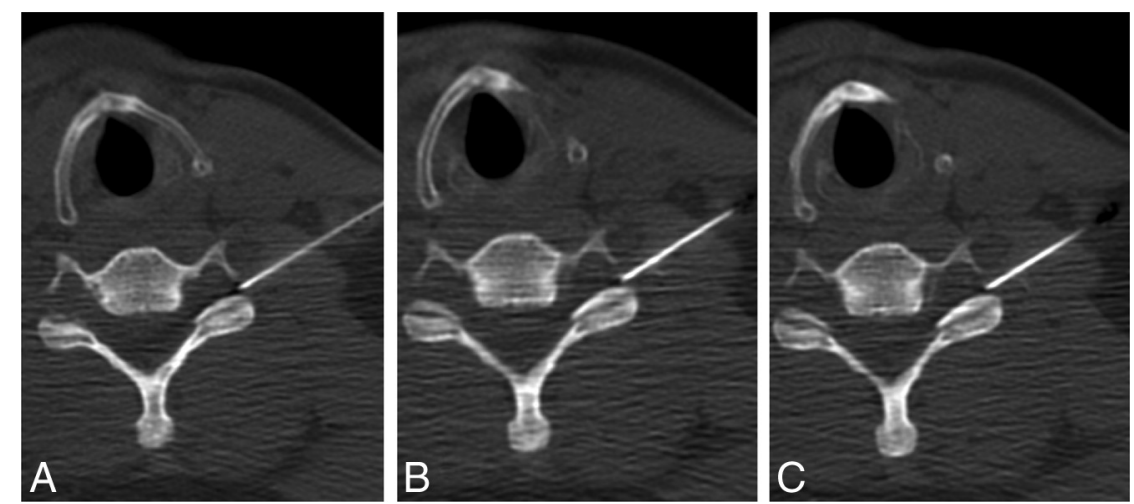

FIG 5. Immediate contrast washout. Preinjection $(A)$, immediate postinjection $(B)$, and delayed postinjection $(C)$ images obtained after injection of $1.0-\mathrm{mL}$ contrast. Neither vascular nor epidural contrast is seen. A preceding injection with $0.2-\mathrm{mL}$ contrast showed similar negative findings, and images obtained cranial and caudal to the needle tip showed no contrast (images not shown).
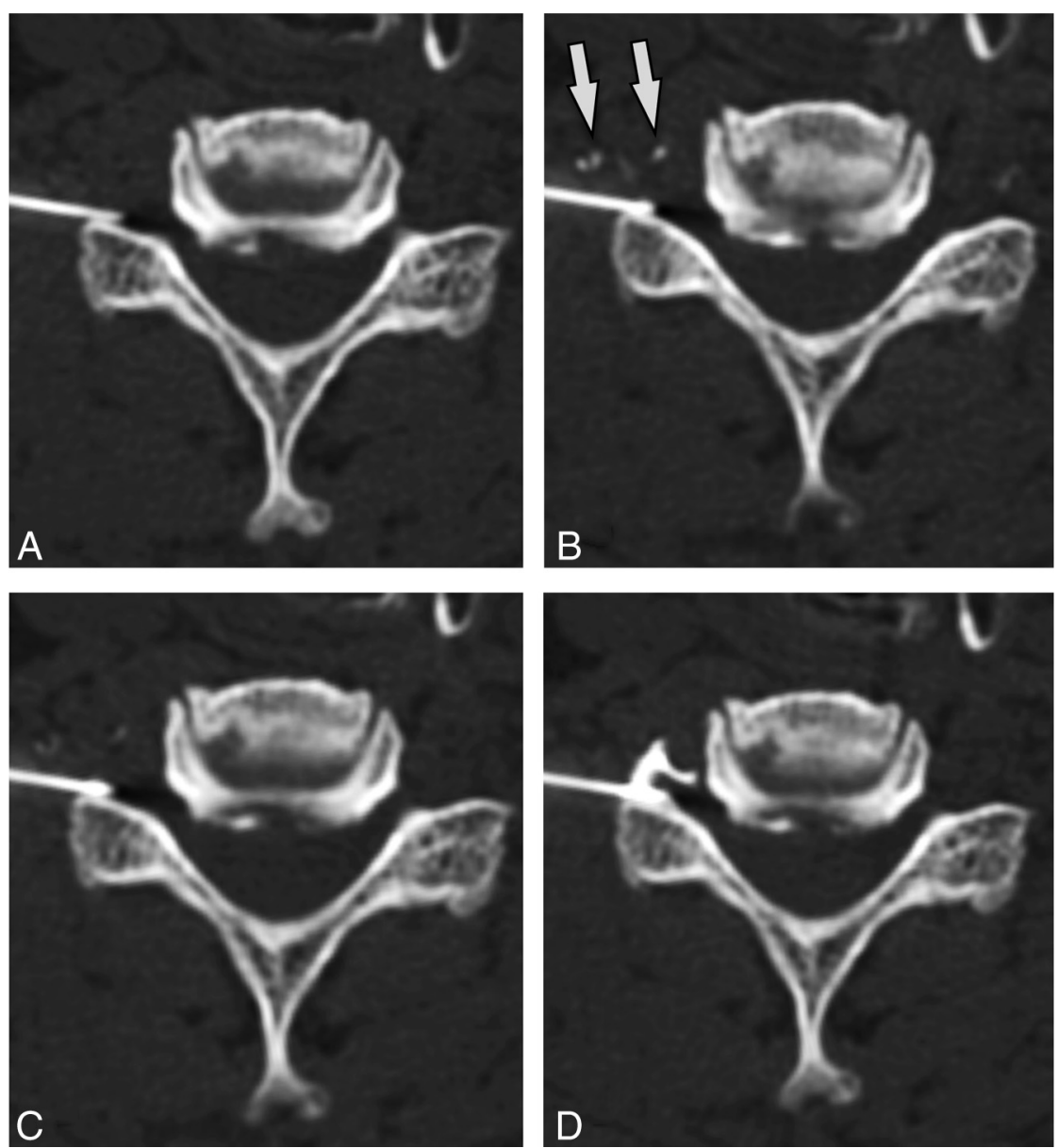

FIG 6. Delayed contrast washout pattern. Preinjection $(A)$, immediate postinjection $(B)$, and delayed postinjection $(C)$ images demonstrate intravascular contrast (arrows) that progressively washes out on delayed images. After needle repositioning $(D)$, re-injection of contrast shows only epidural contrast spread.

in the lumbar region. Furman et $\mathrm{al}^{6}$ found a similar rate of $19.4 \%$ of intravascular injections in a prospective study of 504 cervical TFESIs. In a separate study of 671 lumbar TFESIs, Furman et $\mathrm{al}^{7}$ found a rate of intravascular injection of $11.2 \%$. A recently published prospective investigation by El Abd et $\mathrm{al}^{9}$ of 41 cervical and 113 lumbar transforaminal injections found an incidence of intravascular injection of $17 \%$ during cervical injections and
$15 \%$ during lumbar injections when live fluoroscopy was used. Our study found generally higher rates of detection of intravascular injections during cervical TFESIs (26\%); our rate of intravascular injections during lumbar TFESIs $(8 \%)$ was in the middle of the range of rates reported by other authors.

Relatively less attention has been given in the literature to rates of intravascular injection during ILESI. This may reflect the overall lower incidence of vascular events with this approach and a belief that this approach is less likely to result in a neurologic deficit, given the distance from the radiculomedullary arteries that supply the spinal cord. Although rare, catastrophic outcomes presumed to be vascular in nature have been reported following cervical ILESI. $^{22}$ At minimum, injection into veins during ILESI may decrease the effectiveness of the injection due to decreased epidural deposition of medication. ${ }^{23}$ In a retrospective review of complications associated with $>10,000$ injections, Manchikanti et $\mathrm{al}^{24}$ reported intravascular injections during $4.2 \%$ of cervical and $0.5 \%$ of lumbar epidural injections. Although our study involved considerably fewer patients, our detection rates of intravascular injection during cervical (9\%) and lumbar (2\%) ILESI were higher (Fig 7).

DSA has been advocated by some authors as a way to increase detection of intravascular injection into small arteries, leading to criticism of injections performed without DSA, including those performed by using CTF. Data regarding the actual clinical impact of DSA on rates of catastrophic complications, however, is lacking. Nevertheless, several authors have reported cases of intravascular injections identified with DSA that were missed with live fluoroscopy. ${ }^{25-27}$ One prospective investigation found that the use of digital subtraction angiography identified an additional $2.25 \%$ of cases of vascular injections that would not have been recognized by blood flash, aspiration, or live fluoroscopy, though this study did not specify whether the additional injections identified were venous or arterial. ${ }^{9}$ A separate investigation by McLean et al ${ }^{28}$ reported much higher rates of intravascular injections seen with DSA compared with live fluoroscopy (32.8\% versus $17.9 \%$ ). However, none of the recognized cases in the study, including those seen with both live fluoroscopy and DSA, were judged arte- 
rial. Moreover, reliance on DSA does not preclude the possibility of intra-arterial injection and does not guarantee the absence of significant vascular complications. ${ }^{29}$ Consequently, there is disagreement regarding the role of DSA when performing epidural injections, and this topic is the subject of ongoing, often vigorous debate. $^{30,31}$

Although most vascular injections are most likely venous, most reported studies either do not or are not able to definitively distinguish venous from arterial injections. ${ }^{5}$ In our study, 2 of the 37 cases with positive results were categorized as probably arterial in nature. Although the temporal and inherent spatial resolutions of CT are inferior to those of DSA, the superior contrast resolution of CT allows the detection of small vessels and lets the operator better judge discordance between the volume of injected contrast and the amount of visualized contrast. In our experience, these factors contribute to the detection of small, rapidly flowing vessels, such as arteries, on CT.

Recognition of simultaneous intravascular and epidural injections is potentially important because proceduralists are more likely to miss a vascular injection if an expected epidural contrast pattern is simultaneously visualized. ${ }^{32}$ Previous investigations have reported rates of simultaneous epidural and intravascular injection by using live fluoroscopy of between $15 \%$ and $19 \% .^{8,33}$ In our study, we observed simultaneous epidural and intravascu- lar injection (Fig 8) at much higher rates (32\%) than commonly reported with conventional fluoroscopy.

Despite the successful identification of cases in which vessels were directly visualized at the same time as epidural contrast in our study, we recognize that the cross-sectional nature of CT may impose some limitations as well. For example, purely intravascular injections into vessels that run in the craniocaudal direction (and therefore outside the axial scan plane) would typically be recognized with CTF by the absence of epidural contrast after injection, even if the vessel is not directly seen. However, when simultaneous epidural and intravascular injection occurs involving a vessel oriented in the craniocaudal direction, it is relatively more difficult to recognize, and these injections could potentially be missed in some cases. Nevertheless, our data suggest that the overall rate for detecting intravascular injections, including those that occur concurrently with epidural injections, is not degraded to a major extent by this potential scenario.

Our investigation indicates that attention to several technical aspects of CTF-guided injections can be helpful in facilitating recognition of intravascular injections. First, the use of double-tap intermittent CTF immediately after injection and again 2-3 seconds later to evaluate washout is critical. Washout of vascular contrast was present in all cases, and in most cases, the washout pattern was delayed. If the immediate postinjection image is not obtained, the vessel may never be iden-
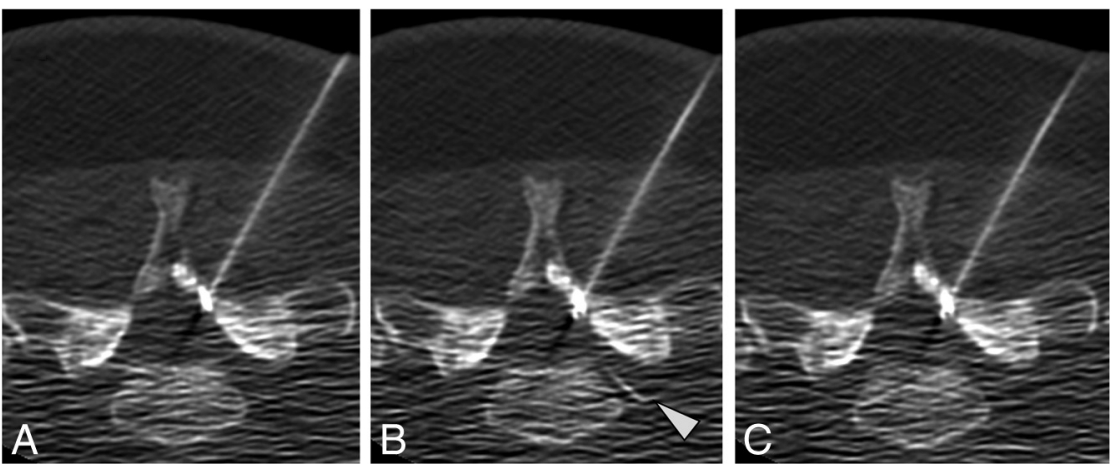
tified because it will be absent on more delayed images. Although the doubletap technique may require a few additional CTF images, the overall increase in dose from these images would be negligible in light of the low tube current and narrow collimation associated with CTF imaging, and any minimal dose increase would be outweighed by the potential safety benefits of identifying intravascular injections. Additionally, it is very important for proceduralists to begin by injecting only a relatively small amount of contrast. This helps prevent

FIG 7. Intravascular injection during cervical ILESI. Preinjection $(A)$, immediate postinjection (B), and delayed postinjection (C) images demonstrate a vessel in the left neural foramen (arrowhead) that washes out on the delayed image.
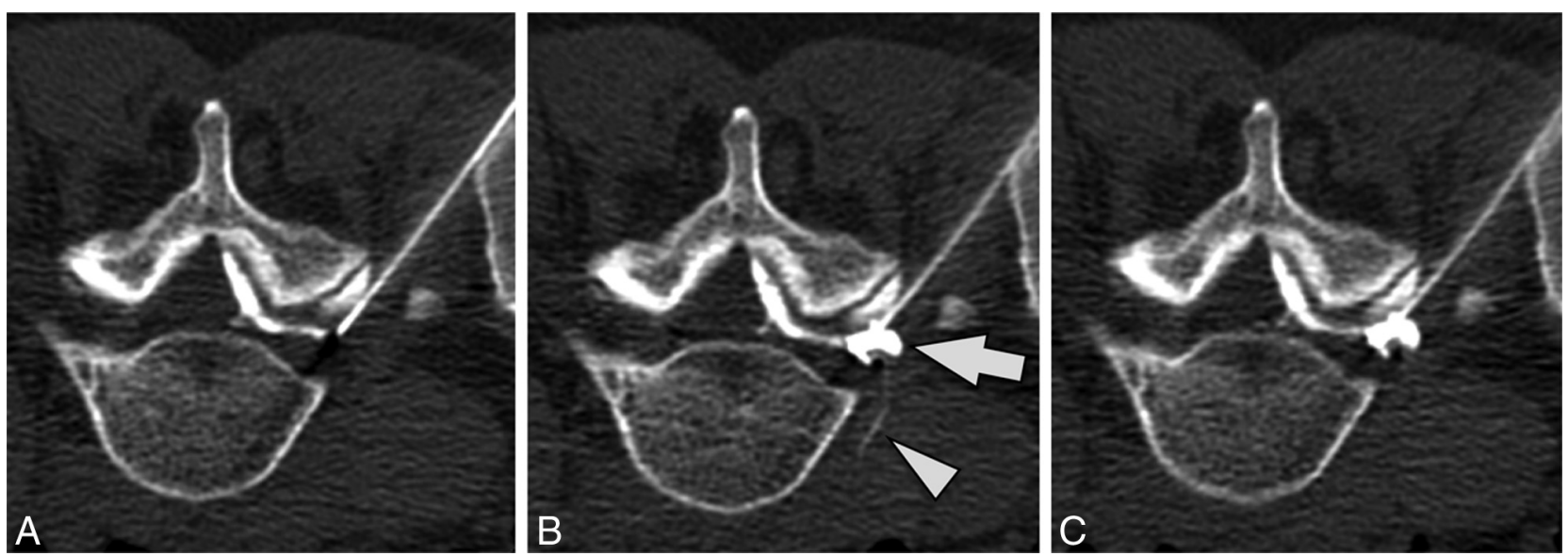

FIG 8. Simultaneous epidural and intravascular injection. Preinjection $(A)$, immediate postinjection $(B)$, and delayed postinjection $(C)$ images demonstrate a vessel extending into the anterior paraspinal soft tissues (arrowhead) that washes out on the delayed image. Epidural contrast in the neural foramen (arrow) persists on the delayed image. Note that there is contrast in the epidural space of the spinal canal on the preinjection image due to prior injection at an adjacent level. 
the vessel from being obscured if there is simultaneous epidural and vascular injection. Proceduralists should also become comfortable with the expected degree of epidural opacification relative to the contrast volume they inject. In some cases, vascular injections will be detected by the absence of expected epidural contrast despite increasing volumes of injected contrast. At the greatest extreme, total or near-total absence of epidural contrast during active injection (assuming intact connector tubing and verification that contrast is actually in the syringe) should indicate that the needle tip is within a high-flow vessel or possibly a vessel running perpendicular to the plane of the image. Finally, the display FOV should be adjusted to include sufficient surrounding soft-tissue structures, including those anterior to the vertebral body, because our data indicate that in some cases, the vessels being opacified with contrast may be remote from the needle tip or even in larger vessels such as the inferior vena cava.

There are several limitations to this investigation. First, and perhaps most important, one can never be sure that all blood vessels, including small-caliber vessels such as radiculomedullary arteries, will be detected when injections are performed under CTF guidance. The ability of CTF to detect some intravascular needle placements, then, should by no means be construed as a guarantee that all intravascular needle placements would be detected. This caveat, however, is also true of injections performed with any imaging technique, including live fluoroscopy and DSA. Additional measures to help mitigate the risk of a vascular event, including the use of nonparticulate steroid and/or an anesthetic test dose, should be considered, particularly for injections with higher risk, such as cervical TFESIs. Second, we compared rates of intravascular injection in our study with previously reported rates with fluoroscopy. Although our data suggest that the rates of detection are generally similar, they do not establish the superiority of one technique versus another, because no direct comparison between modalities was made in this study. The ideal comparison would involve the simultaneous use of CT and fluoroscopy to assess individual injections. Newer technologies such as flat panel CT could potentially facilitate direct comparisons of this type.

Identification of intravascular injection may be related to, but is not the same as, avoidance of significant clinical complications during epidural injections. Because most identified injections are venous and therefore unlikely to result in spinal cord injury or stroke, emphasis should be placed on identifying those techniques that actually reduce complication rates in the clinical setting. Dogmatic assertions or prohibitions regarding the advisability of various injection techniques should be approached cautiously in the absence of such data. Finally, this was a retrospective investigation of a single operator with relatively small numbers of some subtypes of injections. Larger, prospective patient cohorts with multiple proceduralists would be desirable for future investigations.

\section{CONCLUSIONS}

Our investigation demonstrates that intravascular injections can be detected with CT fluoroscopy during epidural injections. Rates of detection of intravascular injection in our study were similar to or greater than rates previously reported for injections performed under conventional fluoroscopy. Technical factors such as the double-tap on CTF following contrast injection, assessment for discordance between the volume of injected contrast and the ex- pected degree of epidural opacification, and maintenance of an appropriate FOV facilitate the detection of such events.

\section{REFERENCES}

1. Manchikanti L, Pampati V, Falco FJ, et al. Assessment of the growth of epidural injections in the Medicare population from 2000 to 2011. Pain Physician 2013;16:E349-64

2. Atluri S, Glaser SE, Shah RV, et al. Needle position analysis in cases of paralysis from transforaminal epidurals: consider alternative approaches to traditional technique. Pain Physician 2013;16:321-34

3. Abbasi A, Malhotra G, Malanga G, et al. Complications of interlaminar cervical epidural steroid injections: a review of the literature. Spine (Phila Pa 1976) 2007;32:2144-51

4. Scanlon GC, Moeller-Bertram T, Romanowsky SM, et al. Cervical transforaminal epidural steroid injections: more dangerous than we think? Spine (Phila Pa 1976) 2007;32:1249-56

5. Nahm FS, Lee CJ, Lee SH, et al. Risk of intravascular injection in transforaminal epidural injections. Anaesthesia 2010;65:917-21

6. Furman MB, Giovanniello MT, O’Brien EM. Incidence of intravascular penetration in transforaminal cervical epidural steroid injections. Spine (Phila Pa 1976) 2003;28:21-25

7. Furman MB, O’Brien EM, Zgleszewski TM. Incidence of intravascular penetration in transforaminal lumbosacral epidural steroid injections. Spine (Phila Pa 1976) 2000;25:2628-32

8. Hong JH, Kim SY, Huh B, et al. Analysis of inadvertent intradiscal and intravascular injection during lumbar transforaminal epidural steroid injections: a prospective study. Reg Anesth Pain Med 2013;38:520-25

9. El Abd OH, Amadera JE, Pimentel DC, et al. Intravascular flow detection during transforaminal epidural injections: a prospective assessment. Pain Physician 2014;17:21-27

10. Kennedy DJ, Dreyfuss P, Aprill CN, et al. Paraplegia following image-guided transforaminal lumbar spine epidural steroid injection: two case reports. Pain Med 2009;10:1389-94

11. Windsor RE, Storm S, Sugar R, et al. Cervical transforaminal injection: review of the literature, complications, and a suggested technique. Pain Physician 2003;6:457-65

12. Smuck M, Fuller BJ, Chiodo A, et al. Accuracy of intermittent fluoroscopy to detect intravascular injection during transforaminal epidural injections. Spine (Phila Pa 1976) 2008;33:E205-10

13. Wagner AL. CT fluoroscopy-guided epidural injections: technique and results. AJNR Am J Neuroradiol 2004;25:1821-23

14. Wolter T, Knoeller S, Berlis A, et al. CT-guided cervical selective nerve root block with a dorsal approach. AJNR Am J Neuroradiol 2010;31:1831-36

15. DePalma MJ, ed. iSpine: Evidence-Based Interventional Spine Care. New York: Demos Medical Publishing; 2011:413

16. North American Spine Society Coverage Committee. Lumbar Epidural Injections: Defining Appropriate Coverage Positions. August 13, 2014. https://www.spine.org/Documents/PolicyPractice/Coverage Recommendations/LumbarEpiduralInjections.pdf. Accessed August 26, 2014

17. Bogduk N. International Spine Intervention Society Practice Guideline For Spinal Diagnositic and Treatment Procedures. San Francisco: International Spine Intervention Society; 2013:409

18. Kranz PG, Abbott M, Abbott D, et al. Optimal contrast concentration for CT-guided epidural steroid injections. AJNR Am J Neuroradiol 2014;35:191-95

19. Guay J. The epidural test dose: a review. Anesth Analg 2006;102:921-29

20. Wagner AL. CT fluoroscopic-guided cervical nerve root blocks. AJNR Am J Neuroradiol 2005;26:43-44

21. Kranz PG, Raduazo PA. Technique for CT fluoroscopy-guided cervical interlaminar steroid injections. AJR Am J Roentgenol 2012; 198:675-77

22. Bose B. Quadriparesis following cervical epidural steroid injections: 
case report and review of the literature. Spine $J$ 2005;5: $558-63$

23. Kaplan MS, Cooke J, Collins JG. Intravascular uptake during fluoroscopically guided cervical interlaminar steroid injection at C6-7: a case report. Arch Phys Med Rehabil 2008;89:553-58

24. Manchikanti L, Malla Y, Wargo BW, et al. A prospective evaluation of complications of 10,000 fluoroscopically directed epidural injections. Pain Physician 2012;15:131-40

25. Yin W, Bogduk N. Retrograde filling of a thoracic spinal artery during transforaminal injection. Pain Med 2009;10:689-92

26. Jasper JF. Role of digital subtraction fluoroscopic imaging in detecting intravascular injections. Pain Physician 2003;6:369-72

27. Verrills P, Nowesenitz G, Barnard A. Penetration of a cervical radicular artery during a transforaminal epidural injection. Pain Med 2010;11:229-31

28. McLean JP, Sigler JD, Plastaras CT, et al. The rate of detection of intravascular injection in cervical transforaminal epidural steroid injections with and without digital subtraction angiography. $P M R$ 2009;1:636-42

29. Chang Chien GC, Candido KD, Knezevic NN. Digital subtraction angiography does not reliably prevent paraplegia associated with lumbar transforaminal epidural steroid injection. Pain Physician 2012;15:515-23

30. Chien GC, Candido KD. Digital subtraction angiography is not the answer for safe epidural injections. Pain Physician 2014;17:E413-14

31. El Abd O, Daud Amadera JE, Pimentel DC, et al. In response to digital subtraction angiography is not the answer for safe epidural injections. Pain Physician 2014;17:E414-15

32. Smuck M, Fuller BJ, Yoder B, et al. Incidence of simultaneous epidural and vascular injection during lumbosacral transforaminal epidural injections. Spine J 2007;7:79-82

33. Smuck M, Tang CT, Fuller BJ. Incidence of simultaneous epidural and vascular injection during cervical transforaminal epidural injections. Spine (Phila Pa 1976) 2009;34:E751-55 